(A)

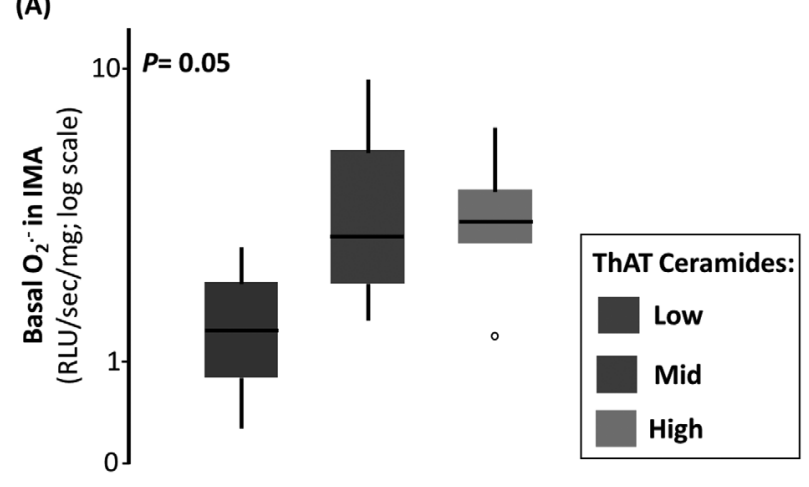

(B)

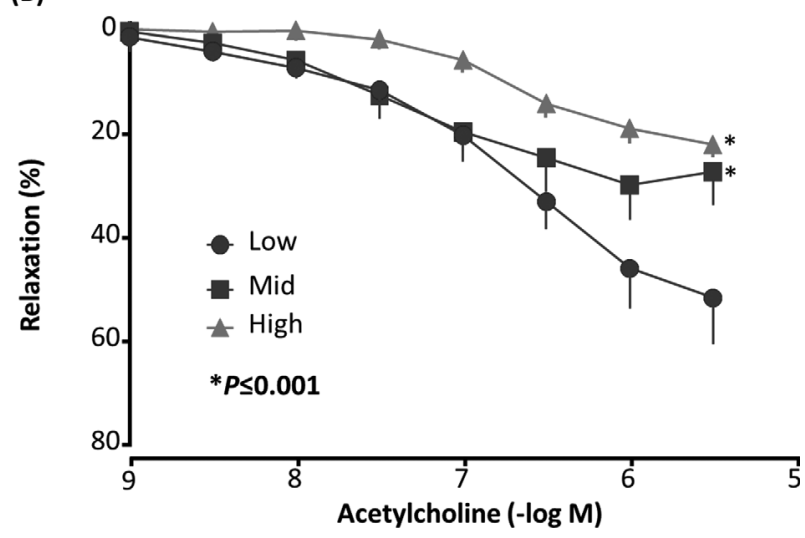

Abstract BS32 Figure 2

\section{BS33 ABSTRACT WITHDRAWN}

\section{BS34 BAV AORTOPATHY EXHIBITS A UNIQUE PATTERN OF AORTIC DEGRADATION EVEN THOUGH THE CLINICAL RISK OF RUPTURE MIRRORS OTHER ANEURYSMS - A MICROMECHANICAL AND MICROSTRUCTURAL APPROACH}

${ }^{1}$ Ya Hua Chim, ${ }^{1}$ Hannah Davies, ${ }^{2}$ Mark Field, ${ }^{1}$ Jill Madine, ${ }^{1}$ Riaz Akhtar*. ${ }^{1}$ University of Liverpool; ${ }^{2}$ Liverpool Heart and Chest Hospital

10.1136/heartjnl-2019-BCS.196

Introduction There have been uncertainties about the appropriate aortic diameter for bicuspid aortic valve (BAV) patients to be used for repairs [1]. On one hand, it is suggested structural weakening of the wall in BAV patients may make these patients more vulnerable to dissection and/or rupture than those with tricuspid aortic valve (TAV). In contrast, the mean aortic diameter for acute type A dissection patients may be greater in BAV patients than in TAV patients. Therefore it is unclear whether BAV patients should be treated differently from those with TAV; we answer the question with our micromechanical and microstructural approach. Here, we measured the micromechanical properties, biochemical properties and elastin microstructural properties of aortic tissue from two aneurysmal groups and a non-aneurysmal group; bicuspid aortic valve with associated aneurysm (BAV-A), idiopathic degenerative aneurysm (DA), and coronary artery by-pass graft (CABG) respectively.

Methods Aortic tissue was obtained from 39 patients (mean age $=62.8 \pm 11.8$ years) undergoing elective surgery. Dynamic nanoindentation was applied to the medial layer using a $100 \mu \mathrm{m}$ flat probe, as reported previously [2]. The same tissues were enzymatically or chemically digested and measured for glycosaminoglycan (GAG) levels using 1-9 dimethylmethylene blue, for collagen using hydroxyproline, and for elastin using fastin elastin kit. To quantify the elastin microstructure, all the tissues were formalin fixed and paraffin embedded, then stained for elastin using Verhoeff Van Gieson. The imaged tissue crosssections were portioned into 10 sections, and quantified for elastin content, number of elastin segments and their lengths. Results The CABG tissues were found to be significantly compliant $(p=0.005)$ relative to BAV-A tissues. However, from the biochemical data, only collagen levels from DA tissues appeared to be significantly higher compared to CABG tissues. BAV-A tissues were found to be significantly $(p=0.048)$ stiffer than DA tissues, while their biochemical levels were the same. CABG and BAV-A tissues had significantly more elastin content for majority of the sections $(\mathrm{p}<0.02)$ relative to DA tissues. DA tissues had low number of segments, and each segment was shorter in comparison to CABG and BAV-A tissues. Although CABG and BAV-A tissues had similar elastin content for majority of the cross-section, from the outer media to adventitia, the aneurysmal tissues had a significant reduction in content $(p<0.04)$. Furthermore, it was noted that there were more elastin segments in BAV-A tissues in comparison to CABG tissues.

Conclusion The elastin microstructures for both aneurysmal groups differ enormously. Our data show that BAV-A tissues are more structurally intact than DA tissues. As current patients with DA have surgical interventions later than patients with BAV-A, we have shown that proposed early surgical interventions may not be necessary for patients with BAV-A. Conflict of interest None declared

\section{BS35 NOVEL INSIGHTS INTO THE USE OF PLATELET RELEASATE AS A THERAPEUTIC APPROACH FOR TISSUE REGENERATION}

${ }^{1}$ Antonios Matsakas*, 'David Scully, ${ }^{1}$ Peggy Sfyri, ${ }^{1}$ Sandrine Verpoorten, ${ }^{1}$ Holly Wilkinson, ${ }^{1}$ Ahmed Aburima, ${ }^{2}$ Laura Gutierrez, ${ }^{2}$ Andrea Acebes Huerta, ${ }^{3}$ Robert Mitchell, ${ }^{3}$ Ketan Patel, ${ }^{1}$ Matthew Hardman. ${ }^{1}$ Hull York Medical School; ${ }^{2}$ Universidad de Oviedo, Spain; ${ }^{3}$ University of Reading

\subsection{6/heartjnl-2019-BCS.197}

Aim Restoration of dysfunctional tissue using platelet-based applications has become an attractive field of research. Due to the cost and safety of such approaches to deliver growth factors in injured tissue; many studies have investigated their potential in regenerative medicine. However, the mechanisms involved in platelet-mediated regeneration are currently poorly understood. The aim of this study was to determine the effect of platelets on cardiac and skeletal myoblast proliferation and differentiation in vitro and establish the role of platelets in skeletal myogenesis ex vivo and in vivo.

Methods Platelet releasate was optimised for improved cellspecific proliferation and differentiation through proliferation assays, immunohistochemical biomarkers, gene and protein expression and proteomics. Mechanistic insights into the effects of platelet secretome interaction with satellite cells, myoblasts, myotubes and myofibres were established through culture with key growth factor inhibitors. Translational aspects of platelet releasate were tested in an in vivo model of injured murine skeletal muscle. Optimisation was next 